\title{
Mediastinal germ cell tumors: many questions and perhaps an answer
}

\author{
J. Wolter Oosterhuis ${ }^{1}$ and Leendert H.J. Looijenga ${ }^{2}$ \\ 'Department of Pathology, Erasmus MC Cancer Institute, Rotterdam, Netherlands. Princess Máxima Center for Pediatric Oncology, Utrecht, Netherlands.
}

\begin{abstract}
Some germ cell tumors (GCTs) in men develop into hematologic malignancies; however, the clonal origins of such malignancies remain unknown. In this issue of the $J C I$, Taylor, Donoghue, et al. unravel the clonal relationship between primary mediastinal nonseminomas (PMNs) and hematologic somatic-type malignancies (HSTMs). Whole-exome sequencing was used to construct phylogenetic trees of the PMNs and the ensuing HSTM clones. HSTMs were derived from multiple distinct clones not detected within the PMNs. Clones from PMNs and HSTMs shared a common precursor, arguably an embryonal carcinoma cell resulting from a reprogrammed primordial germ cell from the thymus. Mutational and copy number variation analysis of a large cohort of patients with PMNs also demonstrated a high prevalence of TP53 mutations not found in testicular nonseminomas. These data likely explain why patients with PMNs are frequently resistant to platinum-based chemotherapy and provide TP53 mutations as potential targets.
\end{abstract}

PGCs that escaped apoptosis after lodging in gonadal and thymic niches as well as the midline of the brain give rise to malignant GCTs. It is in the protective niches that the PGCs, called gonocytes once they arrive in the gonads, could complete their epigenetic reprogramming to late PGCs (9) and progress to the known precursor lesions: germ cell neoplasia in situ (GCNIS) of the testis, gonadoblastoma of the dysgenetic gonad/ovary, and a similar lesion in the thymus (11). The late PGCs/gonocytes progress by default toward seminomas. In contrast, when reprogrammed, a neoplastic gonocyte gives rise to an embryonal carcinoma cell (ECC), which is the malignant counterpart to a naive embryonic stem cell and can give rise to the various histological elements of nonseminomas. Thus, transformed late PGCs/gonocytes determine the malignant behavior of seminomas and nonseminomas, whereas reprogrammed normal early PGCs/gonocytes give rise to the usually benign pediatric GCTs (type I) (Figure 1).

Type II GCTs of the testis, ovary, thymus, and midline of the brain, typically in postpubertal individuals, share histological and genetic features, appearing as pure seminomas or nonseminomas, and possessing gain of the short arm of chromosome 12 and other copy number variations (CNVs). At each anatomic site, the nonseminomas may develop somatic type malignancies, in particular sarcomas and small blue round cell tumors, which may share genetic hallmarks with their somatic counterparts.

\section{The common precursor of PMNs and HSTMs}

In this issue of the JCI, Taylor, Donoghue, et al. (12) assumed that the common precursor of PMNs and HSTMs was most likely a transformed PGC reprogrammed into an ECC within the mediastinum/thymus. ECCs diverged into clones observed in the PMNs, and parallel clones, not detected in the PMNs, formed HSTMs (12), possibly 

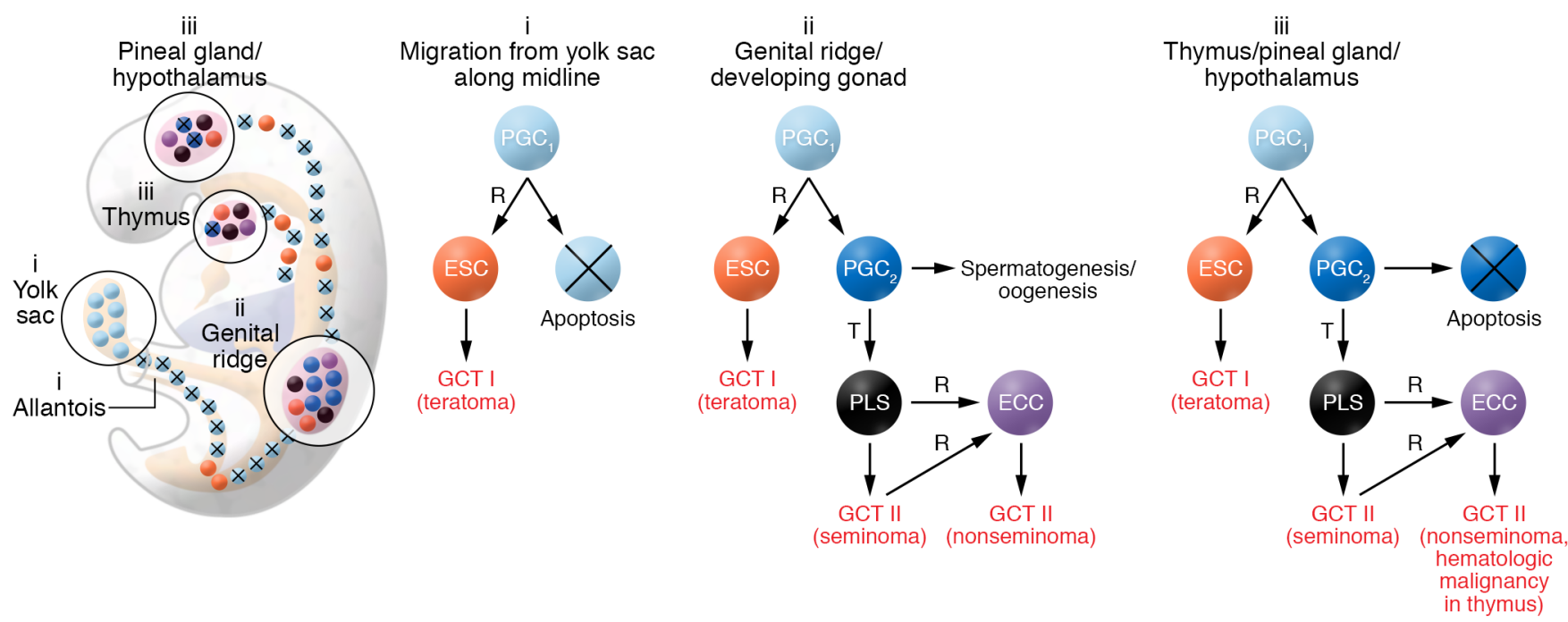

Figure 1. Model for the pathogenesis of type I and II germ cell tumors. PGCs give origin to extragonadal GCTs. During embryogenesis (shown here at week seven), early PGCs (PGC, light blue) migrate from the yolk sac to the genital ridge, and along the midline of the body. Early PGCs undergo apoptosis, unless, very rarely, they are reprogrammed (R) to a primed embryonic stem cell (ESC, orange), the stem cell of type I GCTs, usually benign teratomas. Type I GCTs can also develop in the genital ridge/developing gonads prior to completion of epigenetic reprogramming to late PGCs (PGC , dark blue). Early PGCs escape apoptosis physiologically when they reach the niches in the genital ridge/developing gonad, and nonphysiologically when they lodge in the surrogate niches in the thymus or in the pineal gland/hypothalamus. In each of these niches, early PGCs can complete epigenetic reprogramming to become late PGCs/gonocytes. In the gonads their normal fate is spermatogenesis in the testis and oogenesis in the ovary. Rarely, late PGCs undergo malignant transformation ( $T$ ) and form the precursor lesions for seminoma (PLS), a malignant GCT (black). A transformed late PGC/gonocyte may be reprogrammed into an ECC (purple), which is the malignant counterpart of a naive ESC, and becomes the stem cell that gives rise to the various components of a nonseminoma. In the thymus and the pineal gland/hypothalamus, late PGCs undergo apoptosis, unless they are transformed to the precursors of seminomas. Also, in these sites, transformed PGCs may be reprogrammed to ECCs, giving rise to nonseminomas, and in the thymus also hematologic malignancy.

via a (transient) yolk sac tumor stage. This disparate clonal pattern was not observed in the phylogenetic reconstructions of treatment-resistant testicular nonseminomas (13). However, it is consistent with the results of Dorssers et al. who found that metastases, to which the patient succumbed, were derived from a precursor ECC that was not detected in the primary testicular nonseminoma (14).

An important "bycatch" of Taylor, Donoghue, et al. (12) is the data on mutations in primary mediastinal seminomas and PMNs, which allows comparison with similar data for primary type II GCTs of the testis and brain. Testicular type II GCTs have a low rate of driver mutations, which provide a growth advantage and occur mainly in the KIT/RAS signaling pathway (in about $30 \%$ of seminomas, and $10 \%$ of nonseminomas) $(13,15)$. These KIT and KRAS or NRAS mutations are mutually exclusive, and probably rarely initiating, but rather drive progression $(9,13,16)$, consistent with their increase with age (13). Accordingly, TP53 is virtually always wild type. In intracranial type II GCTs the KIT/
RAS signaling pathway is more frequently mutated $(60 \%)$, and TP53 is mutated in about $5 \%$ (17). Taylor, Donoghue, and researchers (12) report that mediastinal type II GCTs have KRAS or NRAS mutations in 37\% and in TP53 mutations in $61 \%$. When complicated by hematologic malignancies, PMNs show KRAS or NRAS mutations in 63\% and TP53 mutations in 91\%. Neoplastic PGCs may require these high mutation rates to survive within the extragonadal surrogate niches. The high rate of TP53 mutations in PMNs is probably an important factor underlying platinum resistance (18), as loss of function of p53 permits accumulation of CNVs as well as mutations, and interferes with DNA-damage-induced apoptosis. TP53 mutations may also contribute to the high rate of somatic-type malignancies in PMN.

\section{Intriguing questions that remain}

Taylor, Donoghue, and researchers (12) clarify some important issues; however, many intriguing questions remain, such as, why were all the patients evaluated in Taylor, Donoghue, et al. men? This is consistent with the observation that over $95 \%$ of patients with mediastinal type II GCTs are male (11). Why is that the case? The increased risk in males cannot be explained by a higher number of migrating PGCs in males than females, since the incidence of mediastinal type I GCTs, also derived from migrating PGCs, is slightly higher in females than males (16). Being male, so it seems, and more specifically having a Y chromosome, is the most important risk factor for a mediastinal type II GCT. This hypothesis is supported by data on GCT risk in forms of gonadal dysgenesis with and without the Y chromosome. The Y chromosome, more precisely, gonadoblastoma on the Y region (GBY) with the multicopy testis-specific protein Y-linked gene (TSPY), increases the risk of developing a type II GCT up to 70-fold (19, 20). Study of the pathogenesis of GCNIS and gonadoblastoma in dysgenetic gonads and cryptorchid testes incriminates coexpression of OCT3/4 and TSPY for promoting neoplastic transformation of gonocytes in the developing testis (21-23). The same 
mechanism probably operates in the male thymus and midline brain.

Next, why is the risk approximately 70-fold increased in males with Klinefelter syndrome (24)? People with sex-chromosome constitution that results in Klinefelter syndrome, usually XXY, have disturbed spermatogonial niches, such that at puberty virtually no germ cells remain (25), and probably also no GCNIS cells are retained. This deficiency would explain why these individuals are infertile and hardly develop testicular type II GCTs. Conceivably, increased gonadotrophin levels in response to impaired/blocked spermatogenesis enhance the conditions for PGCs lodged in the thymus, increase PGC numbers, and thus the chance of neoplastic transformation (7). Could gonadotrophins, for example, stimulate the production of paracrine factors by thymic epithelial cells, such as KITLG, the key growth factor of PGCs?

Also, why are the type II GCTs of the thymus in people with Klinefelter syndrome exclusively nonseminomas, while in non-Klinefelter patients half of the tumors are seminomas (16)? Perhaps because around puberty PGCs in the thymus degenerate, as with germ cells in the testis. Therefore, even with oncogenic mutations (12), their neoplastic counterparts will not survive unless reprogrammed to an ECC that will develop into a nonseminoma. The reason why seminomas of the brain do exist in Klinefelter syndrome (26) may be that these tumors develop before puberty, before PGCs degenerate.

Last but not least, why do HSTMs in patients with and without Klinefelter syndrome only develop in mediastinal/thymic nonseminomas? Since the HSTMs lack the typical genetic hallmarks of their somatic counterparts, hematologic differentiation is probably not genetically determined (7, 12). Nor does an extragonadal origin per se provide an explanation since midline brain nonseminomas fail to associate with HSTM (16). As suggested before (7), the thymic microenvironment likely favors differentiation of ECCs along hematopoietic lineages.

State-of-the-art comparative (epi) genetic and expression analyses of male and female mediastinal type II GCTs may explain why these tumors are so rare in women, and whether female patients are perhaps mosaic for the $\mathrm{Y}$ chromosome with oncogenic locus/gene (GBY/TSPY). Comparing type II GCTs in males with and without Klinefelter syndrome using similar comparative approaches may reveal why the risk in Klinefelter syndrome is so much increased. Proving the hypothetical role of the thymic microenvironment in establishing hematopoietic lineages in PMN requires experimental testing. Flattened organ cultures (27) of the thymus cocultured with human PGCs (28) might provide a feasible model system, as the thymus may have extended viability, and the culture conditions are easily defined and manipulated.

\section{Conclusions and implications}

Taylor, Donoghue, et al. (12) make two important points, both of which have clinical implications. First, they show that the treatment-refractory HSTMs that may complicate PMNs as yet cannot be diagnosed from the primary tumor. Perhaps the founding clones should be searched for in the bone marrow or blood in highrisk situations, such as PMN in patients with Klinefelter syndrome. Second, the high prevalence of TP53 mutations likely explains the poor prognosis of PMN. Importantly, some of these mutations may be druggable.

\section{Acknowledgments}

We acknowledge Bauke de Jong, whose karyotyping in the 1980 s pointed to the fundamentally different pathogenesis of type I and II GCTs, and the Dutch Cancer Society for supporting our research on GCTs for four decades, enabling us to develop the insights underlying this commentary.

Address correspondence to: J. Wolter Oosterhuis, Prinses Julianalaan 81, 3062 DG Rotterdam, The Netherlands. Phone: 31.6.5183.1946; Email: j.w.oosterhuis@ erasmusmc.nl.

1. Nichols CR, Roth BJ, Heerema N, Griep J, Tricot G. Hematologic neoplasia associated with primary mediastinal germ-cell tumors. $N$ Engl J Med.1990;322(20):1425-1429.

2. Ladanyi M, et al. Cytogenetic and immunohistochemical evidence for the germ cell origin of a subset of acute leukemias associated with mediastinal germ cell tumors. J Natl Cancer Inst. 1990;82(3):221-227.

3. Castedo SM, et al. Cytogenetic analysis of ten human seminomas. Cancer Res. 1989;49(2):439-443.
4. Castedo SM, et al. Chromosomal changes in human primary testicular nonseminomatous germ cell tumors. Cancer Res. 1989;49(20):5696-5701.

5. Dal Cin P, Drochmans A, Moerman P, Van den Berghe $\mathrm{H}$. Isochromosome $12 \mathrm{p}$ in mediastinal germ cell tumor. Cancer Genet Cytogenet. 1989;42(2):243-251.

6. de Bruin TW, et al. Isochromosome 12p-positive pineal germ cell tumor. Cancer Res. 1994;54(6):1542-1544.

7. Zhao GQ, Dowell JE. Hematologic malignancies associated with germ cell tumors. Expert Rev Hematol. 2012;5(4):427-437.

8. Orazi A, Neiman RS, Ulbright TM, Heerema NA, John K, Nichols CR. Hematopoietic precursor cells within the yolk sac tumor component are the source of secondary hematopoietic malignancies in patients with mediastinal germ cell tumors. Cancer. 1993;71(12):3873-3881.

9. Oosterhuis JW, Looijenga LHJ. Human germ cell tumours from a developmental perspective. Nat Rev Cancer. 2019;19(9):522-537.

10. Mamsen LS, Brochner CB, Byskov AG, Mollgard $\mathrm{K}$. The migration and loss of human primordial germ stem cells from the hind gut epithelium towards the gonadal ridge. Int J Dev Biol. 2012;56(10-12):771-778.

11. Williamson SR, Ulbright TM. Germ cell tumors of the mediastinum. In: Marchevsky AM, ed. Pathology of the Mediastinum. Cambridge University Press; 2014:146-168.

12. Taylor J, et al. Evolution of germ cell tumors and shared somatic malignancies from a common precursor. J Clin Invest. 2020;130(12):6668-6676.

13. Taylor-Weiner A, et al. Genomic evolution and chemoresistance in germ-cell tumours. Nature. 2016;540(7631):114-118.

14. Dorssers LCJ, et al. Molecular heterogeneity and early metastatic clone selection in testicular germ cell cancer development. Br J Cancer. 2019;120(4):444-452.

15. Litchfield $\mathrm{K}$, et al. Whole-exome sequencing reveals the mutational spectrum of testicular germ cell tumours. Nat Commun. 2015;6:5973.

16. Oosterhuis JW, Looijenga LHJ. Germ cell tumors from a developmental perspective: cells of origin, pathogenesis, and molecular biology (emerging patterns). In: Nogales FF, Jiminez RE, eds. Pathology and Biology of Human Germ Cell Tumors. Springer; 2017:23-129.

17. Wang L, et al. Novel somatic and germline mutations in intracranial germ cell tumours. Nature. 2014;511(7508):241-245.

18. Bagrodia A, et al. Genetic determinants of cisplatin resistance in patients with advanced germ cell tumors. JClin Oncol. 2016;34(33):4000-4007.

19. Cools M, et al. Impact of the Y-containing cell line on histological differentiation patterns in dysgenetic gonads. Clin Endocrinol (Oxf). 2007;67(2):184-192.

20. Oliveira RM, Verreschi IT, Lipay MV, Eca LP, Guedes AD, Bianco B. Y chromosome in Turner syndrome: review of the literature. Sao Paulo Med J. 2009;127(6):373-378.

21. Cools M, et al. Gonadoblastoma arising in undifferentiated gonadal tissue within dys- 
genetic gonads. JClin Endocrinol Metab. 2006;91(6):2404-2413.

22. Oosterhuis JW, et al. A pathologist's view on the testis biopsy. Int J Androl. 2011;34(4 pt 2):e14-e19; discussion e20.

23. Kaprova-Pleskacova J, et al. Complete androgen insensitivity syndrome: factors influencing gonadal histology including germ cell pathology. Mod Pathol. 2014;27(5):721-730.

24. Hasle H, Mellemgaard A, Nielsen J, Hansen J.
Cancer incidence in men with Klinefelter syndrome. Br J Cancer. 1995;71(2):416-420.

25. Winge SB, Soraggi S, Schierup MH, Rajpert-De Meyts E, Almstrup K. Integration and reanalysis of transcriptomics and methylomics data derived from blood and testis tissue of men with 47,XXY Klinefelter syndrome indicates the primary involvement of Sertoli cells in the testicular pathogenesis. Am J Med Genet C Semin Med Genet. 2020;184(2):239-255.
26. Arens R, Marcus D, Engelberg S, Findler G, Goodman RM, Passwell JH. Cerebral germinomas and Klinefelter syndrome. A review. Cancer. 1988;61(6):1228-1231.

27. Kojima K, et al. Neonatal testis growth recreated in vitro by two-dimensional organ spreading. Biotechnol Bioeng. 2018;115(12):3030-3041.

28. Sasaki K, et al. Robust in vitro induction of human germ cell fate from pluripotent stem cells. Cell Stem Cell. 2015;17(2):178-194. 